\title{
Robust Image Registration via Cepstral Analysis
}

\author{
Ruben Gonzalez \\ Institute for Integrated and Intelligent Systems \\ Griffith University, Gold Coast Campus, QLD, Australia, and \\ Queensland Research Laboratory, NICTA, Australia
}

\begin{abstract}
Cepstrum based image registration has been largely overlooked over the past two decades. Initial investigation of the method was limited to alignment of images exhibiting only translational differences. Later attempts to extend the method to handle rotations proved disappointing. This paper presents a cepstrum-based registration method that provides performance comparable to the state of the art over a wide range of scale factors and rotation angles, with reduced computational load.
\end{abstract}

Keywords; Image Registration; Cepstrum; log-polar transform; Affine Transformation; Global Motion Estimation

\section{INTRODUCTION}

Image registration is the process of aligning two more images of the same scene taken from different perspectives. In the simplest case these images may be displaced relative to each other but they can also exhibit relative scaling and rotation or projective transformation. For many applications that require image data fusion such as creating image mosaics, remote sensing, medical imaging, stereo matching, global motion estimation, and computer vision etc, image registration is a fundamental step. Different image registration techniques have been developed to meet the different constraints and requirements of each of these applications areas.

Registration techniques can be broadly classified into those that characterise the problem as one of feature matching or of correlation. Feature matching methods essentially consist of detecting isolated features in images that are matched with corresponding features across other images to form control points from which a transformation model can be estimated. In practice there are a wide variety of methods to perform each of the steps as surveyed by Brown [1] and Zitova et al. [2]. Alternatively, correlation methods approach the problem by attempting to match image textures (areas) rather than a set of specific features. This matching may be performed using crosscorrelation in the spatial domain, phase-correlation (PC) in the frequency domain or other suitable methods.

Correlation is often preferably performed in the Fourier Domain since using the convolution theorem it is more efficient to perform multiplication in the Fourier Domain rather than cross-correlation in spatial domain. The Fourier Shift property also provides translation invariance. While phasecorrelation by itself only permits translation to be estimated, remapping the images from the Cartesian to log-polar coordinates as in the Fourier-Mellin Transform [3] permits rotation and scale parameters to be estimated from the resulting images by means of phase-correlation. Aside from being in principle robust, phase correlation based techniques are also fast and require constant processing time.

Yet in spite of these advantages, border effects and rotation induced aliasing hampers the effectiveness of FFT based schemes. This limits the ability of these schemes to recover higher scale factors and wide rotation angles [5]. While these unwanted effects can be reduced through windowing and filtering, this removes the image frequencies that these methods require to perform well [4]. Some have even suggested that "FFT based schemes are unable to handle real-world registration problems" [5]. Recent proposed solutions to these problems such as hierarchical, nonlinear, least squares optimization [5] avoids the use of the FFT altogether. Yet while it is robust, it is also fairly slow. A more recent solution making use of normalized gradient correlation (NGC) [6] while slower than phase correlation is considerably faster than least squares optimization while providing comparable performance.

There is however an alternate approach for image registration that has been largely overlooked in the literature. This follows the analogous problem of finding echoes in signals that is typically solved through homomorphic analysis by using the cepstrum [7]. The cepstrum's main appeal is that it requires one third fewer FFTs to be performed than phase correlation. While the cepstrum was first introduced in 1963, [8] its popularity in the signal-processing domain has not extended into that of image registration. Early attempts to apply cepstral analysis to image registration demonstrated that it was limited to image pairs exhibiting only translation and small rotations ( $<2$ degrees) [9]. Most of the work that followed was limited to applications with translational disparities alone, such as optical flow analysis [10], the removal or enhancement of echoes in images [11], determining in conjunction with fuzzy clustering, the translational displacement for registration of multi-sensor images [12]. More recently, Sarnel [13] proposed registration of translated images by applying the cepstrum to the $1-\mathrm{D}$ projections of images along the $\mathrm{x}$ and $\mathrm{y}$-axis. A rotation extended version of the cepstrum making use of histogram stretching, sobel filtering and polar mapping was proposed [14] but the authors demonstrated that it had inferior performance to simple crosscorrelation based methods. No further investigation of the application of 2-D cepstrum for image registration appears in the literature. This paper presents a cepstrum-based approach that is both faster than and gives comparable results to the state of the art image registration methods. 


\section{THEORY}

Let $f(x, y)$ where $[x, y] \in \Re^{2}$ be any digital image, then its 2-D cepstrum, $\hat{f}(x, y)$ is defined as the magnitude of the inverse Fourier transform of the logarithm of the magnitude of the Fourier transform of $f(x, y)$ :

$$
\hat{f}(x, y)=\left|F^{-1}\{\log |\mathcal{F}\{f(x, y)\}|\}\right|
$$

\section{A. Translation Estimation Using Cepstrum}

Let $f_{1}(x, y)$ and $f_{2}(x, y)$ be two digital images where $[x, y] \in \mathfrak{R}^{2}$ that are displaced from each other by an arbitrary translation vector $T=\left[\mathrm{t}_{\mathrm{x}}, \mathrm{t}_{\mathrm{y}}\right]^{\mathrm{T}} \in \mathfrak{R}^{2}$ and attenuated by a given amount. Then $f_{1}$ and $f_{2}$ have the following relationship:

$$
f_{2}(x, y)=a \cdot f_{1}((x, y)+T)
$$

Let $h(x, y)$ be comprised of the sum of $f_{1}$ and $f_{2}$ :

$$
h(x, y)=f_{1}(x, y)+a \cdot f_{1}\left(x+t_{x}, y+t_{y}\right)
$$

Taking the 2D Fourier Transform of both sides of (3) and applying the shift theorem results in:

$$
H(u, v)=F_{1}(u, v)+a F_{1}(u, v) \cdot e^{-j\left(u t_{x}+v t_{y}\right)}
$$

Factoring out $\mathcal{F}_{1}(\boldsymbol{u}, \boldsymbol{v})$ from (4) gives:

$$
H(u, v)=F_{1}(u, v) \cdot\left[1+a e^{-j\left(u t_{x}+v t_{y}\right)}\right\rfloor
$$

Taking the logarithm of both sides of (5) and using the product rule to separate the RHS results in:

$$
\log |H(u, v)|=\log \left|\mathcal{F}_{1}(u, v)\right|+\log \left(1+a e^{-j\left(u t_{x}+v t_{y}\right)}\right)
$$

This expression (6) can be simplified using the Taylor series expansion of $\log (1+\mathrm{x})$ that converges for $|\mathrm{x}|<1$

$$
\log (1+x)=x-\frac{x^{2}}{2}+\frac{x^{3}}{3}-\frac{x^{4}}{4}+\frac{x^{5}}{5}-\ldots
$$

Expanding the second term on the RHS in (6) according to (7) and writing the series as a summation gives:

$$
\log \left(1+a e^{-j\left(u t_{x}+v t_{y}\right)}\right)=\sum_{n=1}^{\infty} \frac{(-1)^{n} a^{n}}{n} \cdot e^{-j\left(u u_{x}+v t_{y}\right)}
$$

Substituting (8) back into (6):

$$
\log |H(u, v)|=\log \left|\mathcal{F}_{1}(u, v)\right|+\sum_{n=1}^{\infty} \frac{(-1)^{n} a^{n}}{n} \cdot e^{-j\left(u t_{x}+v t_{y}\right)}
$$

Applying the inverse Fourier transform to both sides of (9) and substituting for the definition of the cepstrum (1) and applying the Fourier shift theorem one obtains:

$$
\hat{h}(x, y)=\hat{f}_{1}(x, y)+\sum_{n=1}^{\infty} \frac{(-1)^{n}}{n} \delta\left(x-t_{x}, y-t_{y}\right)
$$

The location of the dirac delta given as $\left[\mathrm{t}_{\mathrm{x}}, \mathrm{t}_{\mathrm{y}}\right]^{\mathrm{T}}$ in (10) gives the displacement of $f_{2}$ relative to $f_{1}$.

\section{B. Rotation and Scale Estimation Using Cepstrum}

Let $f_{1}(x, y)$ and $f_{2}(x, y)$ be two images where $[x, y] \in \mathfrak{R}^{2}$ and where $f_{2}$ is a scaled and rotated replica of $f_{1}$ by a scale factor $\mathrm{s}$, and rotation angle $\theta$ such that $f_{1}$ and $f_{2}$ are related by the following transformation:

$$
\begin{array}{r}
f_{2}(x, y)=f_{1}\left(s \cdot x \cos \theta+s \cdot y \sin \theta-x_{o},\right. \\
\text { s.y } \left.\cos \theta-s \cdot x \sin \theta-y_{o}\right)
\end{array}
$$

Taking the Fourier transform of both sides of (11) and using the Fourier translation, scaling and rotation theorems:

$$
\begin{array}{r}
F_{2}(u, v)=\frac{1}{|\mathbf{s}|} \mathrm{e}^{-\mathrm{j}\left(\mathrm{ux}_{\mathrm{o}}+\mathrm{vy}_{\mathrm{o}}\right)} F_{1}(u / s \cos \theta+v / s \sin \theta, \\
u / s \cos \theta-v / s \sin \theta)
\end{array}
$$

Taking the magnitude of both sides of (12) such that $G_{\mathrm{i}}=\left|F_{\mathrm{i}}\right|$ and ignoring the weighting factor $1 /|s|$ gives:

$$
G_{2}(u, v)=G_{1}(u / s \cos \theta+v / s \sin \theta, u / s \cos \theta-v / s \sin \theta)(13)
$$

Denoting the Cartesian coordinates $(u, v)$ on the RHS of (13) in terms of the polar coordinates $(r, \varphi)$ by substituting for $u=r \cos \varphi$ and $v=r \sin \varphi$, one obtains:

$$
\begin{array}{r}
G_{2}(u, v)=G_{1}(r / s \cos \theta \cos \varphi+r / s \sin \theta \sin \varphi, \\
r / s \sin \theta \cos \varphi+r / s \cos \theta \sin \varphi)
\end{array}
$$

Applying the product to sum trigonometric identities gives:

$$
G_{2}(u, v)=G_{1}(r / s \cos (\varphi+\theta), r / s \sin (\varphi+\theta)
$$

Mapping expression (15) into polar coordinates $(r, \varphi)$ where $\boldsymbol{r}=\sqrt{\mathrm{u}^{2}+\mathrm{v}^{2}}$ and $\varphi=\arctan (v / u)$ results in:

$$
G_{2}(r, \varphi)=G_{l}(r / s, \varphi+\theta)
$$

Taking the logarithm of the first variable in (16) permits the division to be converted into a subtraction:

$$
G_{2}(\log r, \varphi)=G_{I}(\log r-\log s, \varphi+\theta)
$$

Substituting $\rho=\log r \& z=\log s$ into (17) gives:

$$
G_{2}(\rho, \varphi)=G_{1}(\rho-z, \varphi+\theta)
$$

The rotation and scaling difference between $G_{1}$ and $G_{2}$ can now be recovered in the polar coordinate system using the cepstrum method as if it were translation in Cartesian space using the method outlined in section 2.1.

\section{CEPSTRUM BASED IMAGE REGISTRATION}

\section{A. Translation only}

To recover the translation vector $\mathrm{T}=\left[\mathrm{t}_{\mathrm{x}}, \mathrm{t}_{\mathrm{y}}\right]^{\mathrm{T}}$ between an $\mathrm{N}$ $\mathrm{x} \mathrm{N}$ image $f_{1}$ and another displaced version $f_{2}$ of the same scene, the two images are superimposed and added and the 2-D cepstrum calculated. The resulting image $\hat{h}(x, y)$ exhibits a strong DC term and other low quefrequency components common to the individual input images as well as a peak at the coordinates representing the location of one image relative to the other. Given a sufficient signal to noise ratio, the recovery of the displacement peak is possible as the highest magnitude quefrequency component after suppressing these common quefrequencies. These may be explicitly suppressed via a high pass filter (19) with a narrow stop band as shown in Fig.1. 


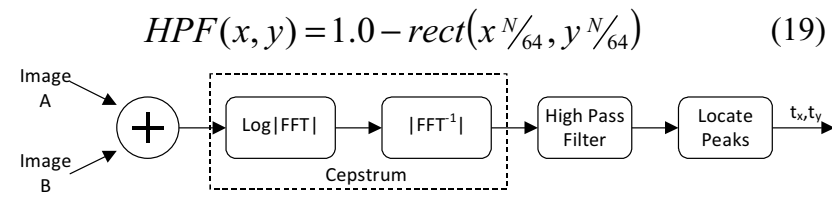

Fig. 1 Translation vector recovery via DC-term removal

Alternatively, improved signal to noise ratios can be obtained by subtracting the cepstrum of one of the two images $\hat{f}_{1}(x, y)$ from the cepstrum $\hat{h}(x, y)$ of the combined images and suppressing the DC term as shown in Fig.2.

$$
\sum_{n=1}^{\infty} \frac{(-1)^{n}}{n} \delta\left(x-t_{x}, y-t_{y}\right)=\hat{h}(x, y)-\hat{f}_{1}(x, y)
$$

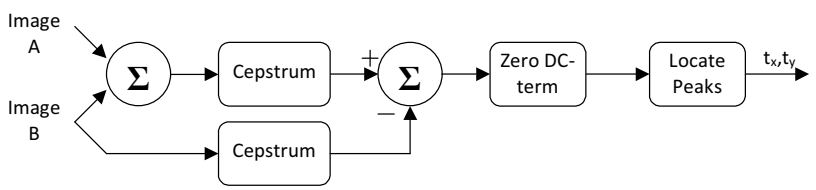

Fig. 2 Translation vector recovery via cepstrum subtraction

In either case, due to the symmetry of the FFT, the cepstral peak will be mirrored about the DC term as can be observed in Fig. 3. Accordingly two translation vectors $T_{1}$ and $T_{2}$ are produced representing $f_{1} \stackrel{T_{1}}{\rightarrow} f_{2}$ and $f_{1} \stackrel{T_{2}}{\leftarrow} f_{2}$.

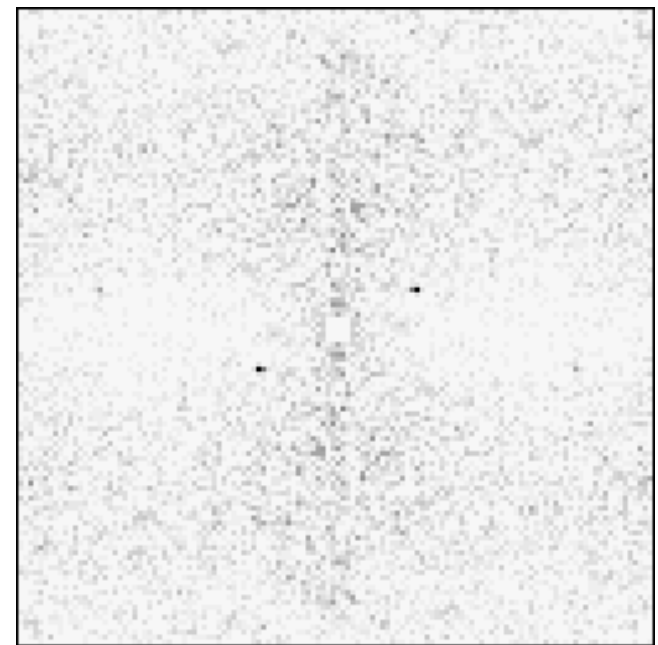

Fig. 3 Cepstral Peaks shown as two dark spots near the centre

Depending on the reference image only one of the two vectors is correct. This ambiguity can be resolved in a variety of ways including inverse transforming image $f_{2}$ according to both translation vectors and then selecting the vector $T_{i}$ producing the smallest residual error $\varepsilon_{i}$ relative to the reference image $f_{l}$ using either the mean square error (MSE) or the sum of absolute difference (SAD) metrics as defined as:

$\varepsilon_{i}=\sum_{x=0, y=0}^{N, N}\left|f_{1}(x, y)-f_{2}\left(x+t_{x}, y+t_{y}\right)-T_{i}\right| ; \quad i=1,2$

\section{B. Rotation and Scaling}

Recovery of the rotation and scaling factors between two images, related to each other as given in (11) requires the source image representations to have the same centre of rotation and scaling, as both are sensitive to the local origin around which they take place. This is achieved by taking the magnitude of the Fourier transform of the sources images as the first step. The source images are then added together and the result remapped into the log-polar domain such that rotation becomes horizontal shifts and scaling becomes vertical shifts.

Following the work reported in [6] the conversion from Cartesian to Log-Polar form is performed using bilinear interpolation by resampling the magnitude of the $\mathrm{N} x \mathrm{~N}$ FFTs on an N/2 x N/2 log-polar grid using the following mapping of a source image $f(x, y)$ in the Cartesian space into the log-polar domain $g(\rho, \varphi)$.

$$
g(\rho, \varphi)=f\left(N / 2+\beta^{y} \cdot \cos (\alpha), N / 2+\beta^{y} \cdot \sin (\alpha)\right)
$$

Where the variables $\boldsymbol{\alpha}$ and $\boldsymbol{\beta}$ are given by:

$$
\alpha=\frac{\pi x}{N} \quad \& \quad \beta=e^{\log (N / 2) / N / 2}
$$

Ideally, the 2-D cepstrum is next calculated and the common quefrequency components suppressed via either (19) or (20). The location of the remaining cepstral peak indicates the rotation and scaling relation between the source images. The basic process is shown in Fig. 5.

The rotation angle $\boldsymbol{\theta}$ and the scale $S$ are recovered as:

$$
\theta=\frac{\pi \varphi}{N} \quad \& \quad S=N^{1 / N^{\rho}}
$$

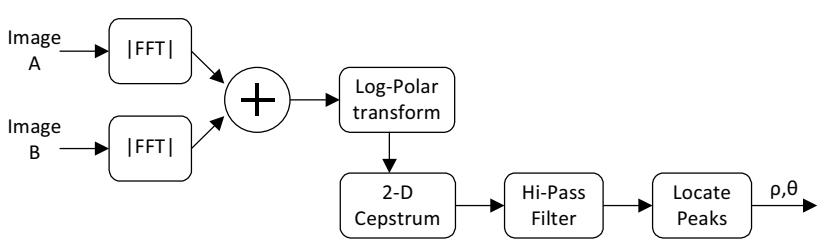

Fig. 4 Basic Scale \& Rotation Invariant Cepstrum Analysis

Simplistically the next step is to use the angle and rotation recovered in (24) to geometrically align the second image and then apply the cepstrum again using the aligned and the reference images to recover any relative translation between them. In practice before this can be performed the polarity of each of the rotation and scaling factors relative to the reference image needs to be resolved. To achieve this, four inverse geometric transformations $( \pm \theta, \pm \mathrm{S})$ need to be applied. Unlike the translation only case, the correct transformation cannot be identified simply as the one giving rise to the least residual error as any unresolved translation will affect the residual.

Accordingly it is necessary to either simultaneously resolve any translation and the polarity of the rotation and scaling factors or to resolve the polarity by using translation invariant representations. Using the Fourier shift property permits the second case to be used by taking the FFT of the four inverse 
transformed images and comparing them to the FFT of the reference image (that was obtained previously). Finally the translation can be recovered as described previously.

\section{Computational Complexity}

Assuming that (19) was used to suppress the DC-term, registration of images that have been rotated, scaled and translated requires a total of ten real Fourier Transforms. Four for obtaining the log-polar cepstrum, another four for resolving the scale and rotation polarity, and two more for recovering the translation vector. This compares favourably with NGC that requires fourteen real and complex FFTs in total, eight for recovering the rotation and scale and another six FFTs for the translation

\section{Enhancing Registration Performance}

In principle the proposed scheme is robust and able to recover arbitrary scale and rotation factors, however in practice a number of matters conspire to reduce the effectiveness of this approach. Spectral leakage due to the image borders at the outset reduces the overall signal to noise ratio. This problem is typically alleviated through the application of a window function. The proposed scheme benefits from windowing of the initial source images and the log-polar transformed image.

Another problem area is due to the frequency distribution of the spectrum of a typical image tending to approximate a Dirichlet kernel centred about zero. Hence, even after zeroing the DC term or high pass filtering, succeeding processes are still heavily influenced by the lower frequency components in an image. Accordingly it is easy for low frequency noise to dominate over higher frequency signals of interest. This is particularly a problem with image registration that relies on the presence of high frequencies in images from sharp edges to accurately fix the location of one image relative to the other. This problem may be alleviated through the use of spectral whitening techniques such as the application of a logarithmic function or taking the square root of the spectral image or by modified unsharp masking.

In the section that follows, the effectiveness of these enhancement methods for improving the performance of cepstrum-based registration are investigated for a wide range of test images. Based on the outcomes of those results the specific details of the cepstral registration algorithm were finalised as:

1. Apply a Hamming Window to images $I_{1}$ and $I_{2}$

2. Obtain $M_{1}=\left|\mathcal{F}\left(I_{1}\right)\right| \& M_{2}=\left|F\left(I_{2}\right)\right|$

3. Obtain $G=\log \left(M_{1}+M_{2}\right)$

4. Using (23), obtain $H$ the log-polar transform of $G$

5. Obtain $\mathrm{J}$ the cepstrum of $H$ using (1)

6. High pass filter $J$ using (19)

7. Apply modified unsharp masking to J using (25)

8. Recover the rotation angle and the scale factor $S$ from the cepstral peak using (24)

9. Obtain the FFT of inverse transformed replicas of $I_{2}$ and resolve the directional ambiguity using (21)

10. Recover the translation component via locating the cepstral peaks in the cepstrum of the sum of $I_{1}$ and the inverse transformed $I_{2}$

\section{EXPERIMENTAL RESULTS}

The performance of cepstral image registration was extensively evaluated using two captured image datasets and another dataset consisting of geometrically transformed replicas of three different 512x512 images. (Lena, goldhill and washsat). Each test image was replicated a total of 9000 times using a different combination of translation, scaling and rotation factors. This resulted in total of 27,000 different image pairs where the scale ranged from 0.0625 to 7.25 in 35 logarithmically uniform steps. The rotation ranged from \pm 180 degrees in 15-degree steps and ten different translation vectors were used based on the scale factors.

\section{A. Generated Dataset Results}

The first experiment using the generated dataset evaluated the performance of the cepstral registration technique using a preliminary Hamming window prior to the log-polar transform, as well as high pass filtering (19) in the first stage to suppress the DC term and other common frequencies prior to the cepstrum calculation and cepstrum subtraction (20).

Of the 27,000 image pairs it was found that only those with scale factors from 0.625 to 1.938 were reliably resolved. For the purpose of these experiments this was determined as those scale factors producing an average error of less than $25 \%$. The value of $25 \%$ was chosen rather arbitrarily since error distribution tends to be a step function: where the scale factors can be recovered the error is typically below $1 \%$ and where they cannot the average error is around $70 \%$.

The average scaling error, across the entire dataset was $63 \%$ and the average rotation error was 62.7 degrees. This baseline algorithm gave results that were comparable to the phase correlation method presented in [3] that was able to recover scale factors up to 1.8 and rotation angles of up to 80 degrees.

The effectiveness of high pass filtering (19) in place of second stage cepstrum subtraction was then evaluated. This slightly improved performance. Scale factors were reliably recovered down to 0.563 and the average scaling and rotation errors for the entire dataset were reduced to $57 \%$ and 57.2 degrees respectively.

The effect of spectral whitening was then investigated in place of the first stage high pass filter through the use of a log function. High pass filtering was used to remove the DC term in the first stage. This further improved the recovery of scale factors to those within the range of 0.375 to 2.56. It also reduced the average scaling error over all possible image pairs down to $45 \%$ and the average rotation error to 45.7 degrees.

In contrast the use of spectral whitening in the second stage negatively impacts the overall performance. This was the case when both logarithmic and milder square root power function were applied. Yet it is clear from inspection of the cepstrum that at higher scale factors that the amplitude of the cepstral peak is reduced relative to that at lower scale factors causing it to be swamped by mid to low frequency noise. To combat this a modified form of unsharp masking was used to emphasize the high frequency components. This entailed subtracting a 
smoothed version of the cepstrum from itself by multiplying the cepstrum with the following filter (27).

$$
\operatorname{HFE}(x, y)=1-0.85 * e^{-4(2 y-N)^{2} / N^{2}}
$$

The application of this filter significantly increased the range of scale factors reliably detected to those within 0.187 to 4.438. The average scale error over all test cases was reduced to $15 \%$ and the average rotation error down to 23.1 degrees.

At this point it was decided to evaluate the sensitivity of the method to the resolution of the log-polar transform by subsampling it by a factor of two. This improved the scaling recovery by $8 \%$ on average but reduced the rotation recovery by $10 \%$. While the range of scale factors recovered improved from 0.187 to 5.06 the rotation factors were less reliably recovered. Also, the resolution dependence of cepstral image registration was evaluated on the same 27,000 pairs of images at reduced resolutions of $256 \times 256$ and $128 \times 128$. As is the case with NGC, the performance was reduced with lower resolution.

\section{B. Comparative Assessment}

The state of the art in image registration is arguably the NGC method that boasts recovery of scale factors up to 6.0. The NGC method has been extensively compared to the well known SIFT method as well as other recent methods such as those described in [5] and [15]. Accordingly the NGC results can be used as a benchmark to assess the performance of cepstrum based image registration relative to not just NGC but also to the techniques that NGC has been assessed against.

To perform an accurate comparative assessment against NGC and the proposed method the same software routines were used to perform functions that were common to both. These included FFT related routines, the log-polar transform and other utility functions. While the implementation of the NGC used here followed the description given in [6], it is likely that the two implementations differ in some small details. For example, single precision floating-point numbers were used in the current implementation. It is likely that any differing numerical results for the NGC method reported from other published ones are due to these differences.

The INRIA dataset [16] and [17] was used to assess the current implementation of NGC and to benchmark the performance of the cepstral method. The results of this assessment are summarised in Table 2 showing the maximum scale factors recovered by each method. The RHS column contains the experimentally obtained results for NGC, with previously reported results in parentheses. As the rotation angles recovered were essentially the same for both methods they have been omitted. Scale is the limiting factor in the performance of both methods. Allowing for rounding errors the experimental results obtained for NGC coincide fairly well with the published ones. The maximum reported scale value reported for NGC could not be replicated in five of the fifteen cases (Belledone, Crolle, Resid, Inria model). There appears to be some error in the reported values for the INRIA case as the ground truth was given as 4.03 and the reported maximum scale recovered was 3.91 whereas the ground truth supplied with the dataset reported a maximum scale factor of 5.88 that was successfully experimentally recovered using NGC. Additionally, while NGC was able to recover the scale factor of
4.35 for the Enismag series of images it was not able to recover lower scale factors of 3.57 or 2.94. Similarly for the Boat series NGC was able to recover a scale factor of 4.35 but not the lower scale factor of 4.01 .

TABle 1 MaXimum SCALE FACTORS RECOVERED

\begin{tabular}{|l|c|c|}
\hline \multicolumn{1}{|c|}{ Name } & Cepstrum & NGC \\
\hline Asterix & 5.78 & $5.78(5.78)$ \\
\hline Belledone & 3.22 & $3.23(5.57)$ \\
\hline BIP & 3.73 & $3.73(3.73)$ \\
\hline Boat & 4.32 & $4.35(4.26)$ \\
\hline Crolle & 3.64 & $2.7(3.97)$ \\
\hline East Park & 5.78 & $5.88(5.78)$ \\
\hline East South & 5.12 & $5.26(5.18)$ \\
\hline Enismag & 4.21 & $4.35(4.76)$ \\
\hline INRIA & 5.78 & $5.88(4.03)$ \\
\hline INRIA model & 3.42 & $4.00(4.82)$ \\
\hline Laptop1 & 6.25 & $6.25(6.22)$ \\
\hline Laptop2 & 1.48 & $1.47(1.51)$ \\
\hline Resid & 4.76 & $4.76(5.85)$ \\
\hline Van Gogh & 3.36 & $3.36(3.38)$ \\
\hline UBC & 2.89 & $2.89(2.89)$ \\
\hline
\end{tabular}

In comparison the cepstral method was able to match the experimental results of NGC in all but one case (Inria Model), and outperformed it in another case (Crolle).

The limiting factor in the recovery of scale factors with the majority of the images in this dataset was the limited range of scale factors available within the dataset itself. Additionally, the scaling factor interval was quite large in most of the images in each series, permitting only a coarse grain performance comparison. Hence, a second dataset was used containing a much wider set of scale factors. This also ensured that the particular sensitivities of each registration method to the specific characteristics of any one dataset did not skew the performance results. The second dataset consisting of 240 photographs [18] was captured with a 6.0 mega pixel, 12x optical zoom Minolta DiMAGE Z6 digital camera. Each series of images in this dataset contained a range of rotation angles and scale factors exceeding 10:1. Having an average number of 25 images in each series provided an average scaling increment of about $10 \%$ for each image. The images were sub sampled to $1024 \times 768$. The results using this dataset are given in Table 2. The maximum ground truth scale factor for each image series is given in parenthesis following the image name.

Table 2 Maximum Scale Factors Recovered for Second Dataset

\begin{tabular}{|l|c|c|}
\hline Name & Cepstrum & NGC \\
\hline Painting (11.1) & $\mathbf{5 . 5 1}$ & 3.57 \\
\hline Tractor (11.1) & 6.00 & $\mathbf{6 . 2 5}$ \\
\hline Crane (11.4) & 6.22 & 6.22 \\
\hline HiRise (11.2) & $\mathbf{4 . 8 2}$ & 3.47 \\
\hline TwoLights (11.1) & $\mathbf{4 . 1 1}$ & 2.49 \\
\hline Plant (10.8) & $\mathbf{5 . 4 4}$ & 4.21 \\
\hline Steps (10.9) & 4.59 & 4.59 \\
\hline Tower $(16.0)$ & $\mathbf{3 . 7 3}$ & 3.18 \\
\hline Uni & 5.31 & 5.31 \\
\hline Vending (10.6) & 6.07 & 6.07 \\
\hline
\end{tabular}


Using this dataset, the cepstrum method outperformed NGC in five of the tests. In contrast NGC outperformed the cepstrum only on one occasion (Tractor see Fig.7) and both performed equally on the remaining four tests. Neither method recovered the full range of available scale factors.

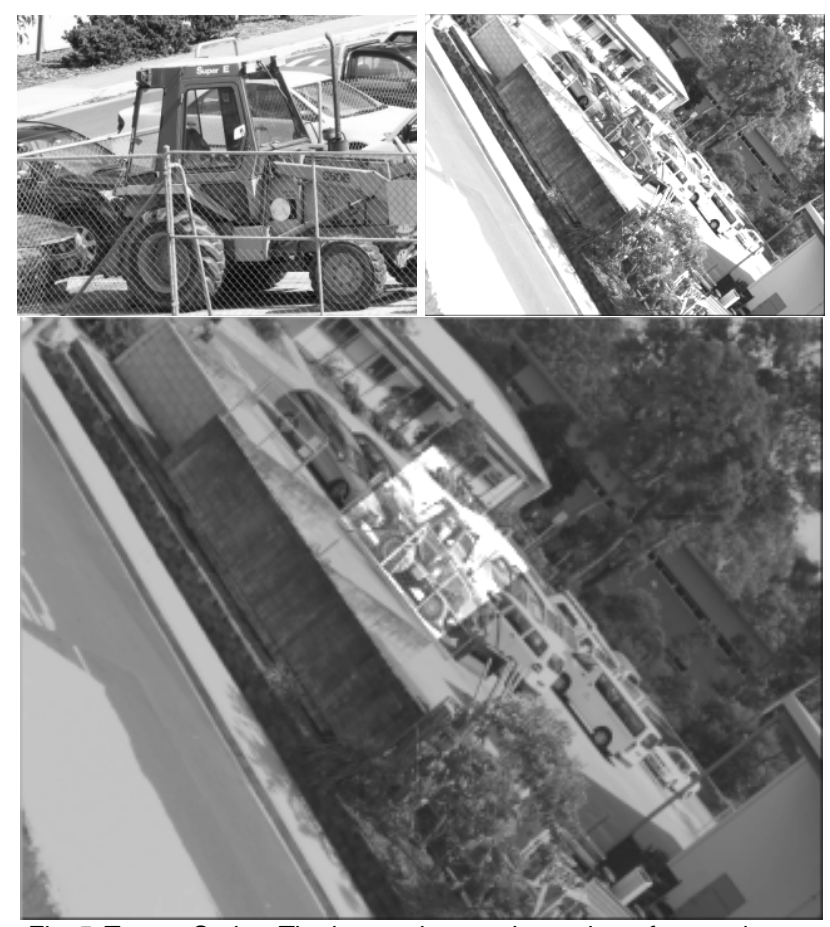

Fig. 5. Tractor Series. The bottom image shows the reference image rotated, scaled, translated and superimposed over the reference image, according to the parameters recovered using the cepstrum.

\section{CONCLUSION}

While the cepstrum has been largely overlooked as a technique for image registration for the past two decades, appropriately deployed, it can competently compete against state of the art image registration methods. Not only can it recover the arbitrary rotation and a relatively wide range of scaling factors but with a reduced computational burden. It requires fewer FFTs, (all of them real) than other state of the art methods. It accordingly lends itself to application in environments with limited computational resources, be that memory or processing capability. The cepstrum also shares the benefits common to other Fourier-based methods; constant processing time and robustness to noise.

\section{REFERENCES}

[1] L.G. Brown. "A survey of image registration techniques." ACM Computing Surveys, vol.24, no.4 pp.325-376, Dec. 1992.

[2] B. Zitová, J. Flusser: "Image registration methods: a survey." Image Vision Computing, vol. 21, no.11, pp.9771000, 2003.

[3] B.S. Reddy, B.N. Chatterji, "An FFT-based technique for translation, rotation, and scale-invariant image registration", IEEE Transactions on Image Processing, vol.5, no. 8, pp.1266-1271, 1996.

[4] H.S. Stone, B. Tao, M. Mcguire, "Analysis Of Image Registration Noise Due To Rotationally Dependent Aliasing" Journal of Visual Communication and Image Representation - JVCIR, vol. 14, no. 2, pp. 114-135, 2003

[5] S. Zokai, G. Wolberg, "Image Registration Using Log-Polar Mappings for Recovery of Large-Scale Similarity and Projective Transformations." IEEE Transactions on Image Processing, vol.14, no.10, pp. 1422-1434, 2005.

[6] G. Tzimiropoulos, V. Argyriou, S. Zafeiriou, T. Stathaki, "Robust FFT-Based Scale-Invariant Image Registration with Image Gradients," IEEE Trans. on Pattern Analysis and Machine Intelligence, vol.32, no.10, pp. 1899-1906, Oct. 2010

[7] D.G. Childers, D.P.Skinner, R.C. Kemerait, "The cepstrum: A guide to processing," Proceedings of the IEEE , vol.65, no.10, pp. 1428-1443, Oct. 1977

[8] B. P. Bogert, M. J. R. Healy, and J. W. Tukey: "The Quefrency Alanysis of Time Series for Echoes: Cepstrum, Pseudo Autocovariance, Cross-Cepstrum and Saphe Cracking". Proceedings of the Symposium on Time Series Analysis (M. Rosenblatt, Ed) Chap.15, pp.209-243. 1963.

[9] DJ Lee, TF Krile, and S Mitra, "Power cepstrum and spectrum techniques applied to image registration", Applied Optics, vol. 27, pp.1099 - 1106, 1988.

[10] E. Bandari; J. Little, "Spatial-quefrency approach to optical echo analysis," Computer Vision and Pattern Recognition, 1992. Proceedings CVPR '92., 1992 IEEE Computer Society Conference on , vol., no., pp.850-852, 15-18, June 1992

[11] J.K. Lee, M. Kabrisky, M.E. Oxley, S.K. Rogers, D.W. Ruck, "The complex cepstrum applied to two-dimensional images", Pattern Recognition, Vol., 26, no.10., pp.1579-1592, 1993.

[12] G. Zamora, M. Dickens, S. Mitra, "A robust registration technique for multi-sensor images," 1998 Image Analysis and Interpretation, IEEE Southwest Symposium on, vol., no., pp.87-90, 5-7 April 1998

[13] H. Sarnel, "A robust cepstrum-based algorithm for image registration using projections," Signal Processing, 2002 6th International Conference on, vol.1, no., pp. 813- 816 vol.1, 26-30 Aug. 2002

[14] T. M. Lehmann, C. Goerke, A. Kaupp, W. Schmitt, R. Repges, "A Rotation-extended Cepstrum Technique and Its Application to Medical Images", Pattern Recognition and Image Analysis, Vol. 6, No. 3, pp. 592-604, 1996.

[15] W. Pan, K. Qin, and Y. Chen, "An Adaptable-Multilayer Fractional Fourier Transform Approach for Image Registration," IEEE Trans. Pattern Analysis and Machine Intelligence, vol. 31, no. 3, pp. 400-413, Mar. 2009.

[16] Image Database, http://www.robots.ox.ac.uk/vgg/research/affine/, 2010.

[17] Image Database, http://lear.inrialpes.fr/people/mikolajczyk/, 2010.

[18] Image Database, http://www.griffith.edu.au/professional-page/rubengonzalez 